\section{$\$$ Research Square}

\title{
A Putative Tumor Suppressing Role of hsa-miR-154 in Breast Cancer that acts by Targeting CLOCK Gene
}

\section{Eiman Ibrahim}

Yale University School of Medicine

\section{Alison Fritz}

Yale University School of Public Health

Alan N Fu

Yale University School of Public Health

\section{Barbara E Ehrlich}

Yale University School of Public Health

\section{Yong Zhu ( $\sim$ Yong.Zhu@yale.edu )}

Yale University School of Public Health https://orcid.org/0000-0002-6957-1114

\section{Research Article}

Keywords: hsa-miR-154, breast cancer, expression, survival, CLOCK gene

Posted Date: March 11th, 2021

DOl: https://doi.org/10.21203/rs.3.rs-278396/v1

License: (c) (1) This work is licensed under a Creative Commons Attribution 4.0 International License. Read Full License 


\section{Abstract}

\section{Background}

MicroRNA-154 (hsa-miR-154) is a novel miRNA. Emerging information shows that hsa-miR-154 participates in multiple physiological and pathological processes and is generally identified as a tumor suppressor in multiple types of cancers. Methylation of the hsa-miR-154 gene could also be altered by circadian disruption associated with night shiftwork.

Results

In this study, we tested whether hsa-miR-154 expression is downregulated in breast cancer and whether hsa-miR-154 targets any circadian genes. Using publicly available datasets and bioinformatics analysis, we first demonstrated that expression levels of hsa-miR-154 were significantly lower in breast tumors compared to normal breast tissues and its expression correlated with clinical outcomes. Downregulation of hsa-miR-154 was also confirmed in breast cancer cell lines and restoration of hsa-miR-154 by transfection significantly inhibited growth of these cells. Further bioinformatics screening indicated that the circadian gene CLOCK is likely a hsa-miR-154 target.

\section{Conclusions}

These findings suggest a potential tumor suppressing role of hsa-miR-154 in breast cancer that acts by targeting CLOCK gene. Exploration of hsa-miR-154 for its anti-cancer effect may provide information on its potential for therapeutic application.

\section{Introduction}

MicroRNAS (miRNAs) is a class of endogenous small non-coding RNAs negatively regulating gene expression by inducing degradation or translational inhibition of target mRNAs. These miRNAs play an important role in tumorigenesis via control of genes in cancer related cellular processes including cell proliferation, differentiation, and apoptosis (1). Aberrant miRNA expressions have been observed in a variety of cancers, suggesting that they may function as either oncogenes or tumor-suppressor genes in tumorigenesis (2).

Hsa-miR-154, located on chromosome 14q32, has been detected in recent publications to be downregulated in several cancer types such as lung cancer and multiple myeloma (3-5). Its tumor suppressing role has been suggested by in vitro functional assays (6-8) and potential targeted oncogenes ADAM metallopeptidase domain 9 (ADAM9) (9) and E2F transcription factor 5 protein (E2F5) (10). Few studies have examined the role of hsa-miR-154 in breast cancer.

Expression of miRNAs can be affected by many factors including environmental exposures, which may result in adverse health effects (11). In our previous study, we performed an epigenome-wide analysis and detected DNA methylation changes in the promoter of several miRNAs including hsa-miR-154 among 
night shift workers (12) and night shiftwork has been classified as a probable cause of cancer in humans by The International Agency for Research on Cancer (IARC) (13). There is also compelling evidence to suggest that miRNAs play essential roles in the fine tuning of rhythmic outputs from the central circadian clock (14-16). Furthermore, the core circadian genes play a significant role in breast cancer tumorigenesis, possibly by acting on hormone regulation or other cancer related pathways (17).

In the current study, we tested the hypothesis that hsa-miR-154 has a tumor suppressing role in breast cancer by targeting oncogenic circadian genes. We compared its expression between breast tumor and normal tissues, correlated its expression to breast patient survival and predicted its circadian gene targets.

\section{Materials And Methods}

Hsa-miR-154 expression in breast cancer tissues. We explored hsa-miR-154 sequence abundance in breast cancer tissues. For that purpose, we used the YM500 database (18) which contains more than eight thousand small RNA sequencing (smRNA-seq) data sets and provides integrated analysis results for several cancer miRNome studies via multiple interactive interfaces (Expression, Novel miRNAs, isomiRs and thousands of smRNA-Seq datasets). The YM500 database also provides information about miRNA isoforms and arm switching discovery, however, there are limited miRNA editing analyses in different tissues and cancer types.

We also analyzed the hsa-miR-154 data available through the OncomiR Cancer Database (OMCD)( www.oncomir.umn.edu/omcd) (19). OMCD was developed at the University of Minnesota. This database was designed to allow systematic comparative genomic analyses of miRNA sequencing data derived from > 9500 cancer patients tissue samples available in the Cancer Genome Atlas (TCGA). Data were checked for equal variance and normal distribution via $\mathrm{F}$ test and Shapiro Wilk tests respectively in $\mathrm{R}$ Studio. Statistical comparisons between two groups were conducted by unpaired Student's t-test; oneway analysis of variance (ANOVA) was performed when the dataset contained more than two groups. $P<$ 0.05 was considered to be statistically significant.

Hsa-miR-154 expression and survival of breast cancer patients. To explore the effect of hsa-miR-154 expression on the survival rate of breast cancer patients, we used the Kaplan Meier plotter (KMplotter) tool (20). Data of solid tumors including lung, liver, pancreatic, ovarian, gastric, and breast cancers are available in this database. Cohorts of patients were split by median expression values through auto select best cut-off. Clinical data, including human epidermal growth factor receptor 2 (HER2), estrogen receptor, progesterone receptor status, lymph node status, tumor pathological grade, intrinsic subtype were displayed. A database was established using gene expression data and survival information of 2622 patients. The KMplotter MiRNA gene expression datasets used in the study were downloaded from gene expression omnibus (GEO) (http://www.ncbi.nlm.nih.gov/geo/), TCGA (http://cancergenome.nih.gov/), European genome-phenome archive (EGA) (https://ega.crg.eu/), and PubMed (http://www.pubmed.com). 
Cell lines and cell culture. Human breast cancer cells MCF-7 and normal breast epithelial cells MCF-10A were purchased from the American Type Culture Collection (ATCC) (Manassas, VA, USA). MCF-10A cells were maintained in MEGM (Life Technologies, CA, USA) supplemented with $100 \mathrm{ng} / \mathrm{mL}$ cholera toxin. MCF-7 cells were cultured in DMEM (Life Technologies, CA, USA) supplemented with $10 \%$ fetal bovine serum, $1 \%$ penicillin-streptomycin. The cells were cultured at $37^{\circ} \mathrm{C}$ in a humidified atmosphere consisting of $5 \% \mathrm{CO}_{2}$. The culture medium was changed once every 2 days.

RNA isolation and detection of Hsa-miR-154. Total RNA was isolated from two human breast tissue cell lines (MCF7 as breast cancer) and (MCF10A as a non-cancer) using the miRNeasy Mini Kit (reference 217004, Qiagen, CA). A complementary DNA (CDNA) conversion for each of the cell lines was done using an NCode kit (Invitrogen ${ }^{T M}$ MIRQ100). To determine levels of mature micro RNA, polyadenylated mature micro RNA sequences were first generated and converted to cDNA using the NCode miRNA First-Strand cDNA Synthesis Kit (Invitrogen). The cDNA was then amplified using a custom hsa-miR-154 specific forward primer. Sequences for the forward and reverse primers used to detect hsa-miR-154 precursors were 50- AGC AGC ATT GTA CAG GGC TAT CA-30 and 50 - TAG GTT ATC CGT GTT GCC TTC G-30 respectively. A universal reverse primer targeting on the polyadenylated region of the miRNA was also used. Mature miRNA levels in cancer cell lines relative to normal cells were assessed using the delta delta CT (2- $\Delta \Delta \mathrm{CT})$ method with normalization to U6B in which $\Delta \Delta \mathrm{CT}=$ (CT miRNA - CT U6) target - (CT miRNA - CT U6) control (21). All quantitative PCR reactions were conducted in triplicate on an $A B I 7500$ Fast Real-Time PCR instrument (Applied Biosystems) using the QuantiFast SYBR Green PCR Kit (QIAGEN). The qPCR was run in two stages. Initial denaturation was 3 min at 95 degrees Celsius. Denaturation was 40 cycles of 3 seconds at 95 degrees Celsius and annealing and elongation (data collection) was 40 cycles of 30 seconds at 60 degrees Celsius. A dissociation step was added to check for primer-dimer activity.

Cell proliferation assay. A 4-day hsa-miR-154 transfection cell proliferation assay was done in triplicate for both the MCF-7 breast cancer cell line and MCF-10a normal breast cell line. MCF-7 cells were cultured in Dublecco's Modified Eagle Medium supplemented with 10\% heat-inactivated Fetal Bovine Serum. MCF10-a cells were cultured with Mammary Epithelial cell Growth Medium. Cells were transfected with a hsa-miR-154 mimic, or a scrambled negative control (Qiagen) using the Lipofectamine RNAiMAX transfection reagent (Invitrogen), according to the manufacturer's protocol. Briefly, $4 \mu$ l of $10 \mu \mathrm{M}$ hsa-miR154 mimic, or negative control was mixed with $0.1 \mu \mathrm{l}$ of RNAiMAX reagent in $20 \mu \mathrm{l}$ of OPTI-MEM (Invitrogen) and was used for each well. The complex was then added into a 98-well plate and incubated for 20 minutes at room temperature. Approximately $100 \mu \mathrm{l}$ of $30 \mathrm{cells} / \mu \mathrm{l}$ were then seeded into each well dish for a total volume of $120 \mu \mathrm{l} .20 \mu \mathrm{l}$ of MTS solution per well was then added in the dark room hood and incubated for 2 hours at $37^{\circ} \mathrm{C}$ before measuring a baseline cell concentration. MTS was similarly added to a new row every day for 4 days.

Bioinformatic search for Hsa-miR-154 binding sites in circadian genes. A target screening was completed using miRecords software to observe likely interactions by screening potential untranslated region (UTR) binding sites between 58 miRNA identified in the differential screen and the ten core circadian clock 
genes CLOCK, CRY1, CRY2, PER1, PER2, PER3, BMAL1, NPAS2, CSNK1E and TIMELESS (22). Seven target prediction algorithms were originally used to obtain the results: DIANA-microT, miRanda, MirTarget2, PicTar, PITA, NB MiRTar, Targetscan, and RNAhybrid. Prospective binding sites were predicted according to the number of base pair matches to the 50 miRNA seed region, the degree of compensatory 30 nonseed matches, and the number and nature of mismatched pairs (miRanda, TargetScan/TargetScanS, and PITA), as well as thermodynamic stability (miRanda and RNAhybrid). Results from RNAhybrid were excluded from the dataset because they were almost universally positive. Of the identified 58 micro RNAs, 12 were not recognized by the miRecords database.

\section{Results}

\section{Downregulation of hsa-miR-154 in cancer tumor tissues}

Using YM500 database, we found the expression profiles of hsa-miR-154 in breast cancer tissues based on $>10,000$ cancer-related RNA seq data sets and $>3000$ more small RNA-seq data from TCGA, which included database 1006 primary tumors, 103 normal breast tissues, 6 metastatic tissues. Figure 1a showed that the expression hsa-miR-154 was downregulated in breast tumor tissues compared to normal breast tissues. Because raw data was not available from YM500 database for $\mathrm{p}$ value calculation, we downloaded miRNA sequencing data of 782 breast cancer tissues and 87 normal tissues in the OncoMir database for comparison. Our analysis showed that hsa-miR-154 is significantly downregulated in breast cancer tissues $(P=0.0019)$. The findings indicate that hsa-miR-154 serves as a potential tumor suppressor that might inhibit the pathogenesis of breast cancer (Figure 1b).

\section{Downregulation of hsa-miR-154 associated with poor survival of breast cancer patients}

The survival analysis included 4 different patient datasets, 1262 patients from the Molecular Taxonomy of Breast Cancer International Consortium (Metabric), 1078 patients in the TCGA, 181 patients in the GSE40267, and 101 patients in the GSE19783 of the Gene Expression Omnibus. A Kaplan-Meier plot was generated to assess the effect of hsa-miR-154 on breast cancer prognosis in a different dataset (Metabric), as shown in Figure 1c. High hsa-miR-154 expression correlated with better survival in METABRIC breast cancer patients $(P=9.0 \mathrm{e}-6$, false discovery rate $(F D R)=1 \%$, hazard ratio $(H R)=0.46$ (0.53-0.78) These results indicated that high hsa-miR-154 expression was a favorable independent prognostic factor for breast cancer patients.

\section{Hsa-miR-154 inhibits cell proliferation of breast cancer cells}

RT-PCR revealed significantly lower endogenous hsa-miR-154 levels in MCF-7 cells compared to MCF10a cells $(P<0.001)$ (Figure 2a). Hsa-miR-154 transfection in both MCF-7 and MCF-10a cell lines significantly inhibited the proliferation and slowed growth (Figure $2 \mathrm{~b}$ ). The control grew approximately twice as fast in both cell lines when transfected with hsa-miR-154. At 96h, hsa-miR-154 had less effect slowing MCF-10a growth than it did on MCF-7 growth (adjusted $\mathrm{P}=0.016$ after multiple comparison correction). 


\section{CLOCK gene is a putative target of hsa-miR-154}

In general, a miRNA is determined to be a likely target if there are four or more likely interactions. Using the DIANA-microT (23) , miRanda , MirTarget2, PicTar, PITA, NB MiRTar, and Targetscan target prediction algorithm, the CLOCK gene was identified as a likely target for hsa-miR-154 in 5 out of 9 tools. Figure $3 a$ illustrates the hsa-miR-154 identifications using several tools.

\section{Discussion}

Results from this study indicated that hsa-miR-154 was downregulated in breast tumor tissues and low expression of hsa-miR-154 was associated with poor breast cancer survival. A functional proliferation assay further confirmed that restoration of hsa-miR-154 significantly decreased cell growth in MCF-7 breast cancer cells. These results were consistent with previous findings that hsa-miR-154 was downregulated in many cancer types including colorectal cancer (24) and osteosarcoma (25). Previous studies also showed that restoration of intracellular hsa-miR-154 inhibited tumorigenesis and $\mathrm{G}_{1} / \mathrm{S}$ transition in the liver cancer cells $(26)$ and breast cancer cells $(9,10)$. These findings suggest that hsamiR-154 serves as a potential tumor suppressor.

The circadian gene CLOCK was predicted as a potential target of hsa-miR-154 in our study. CLOCK is a transcriptional regulator that mediates the expression of numerous cancer-related genes and plays an extensive regulatory role in DNA repair and other cancer relevant pathways (27). Genetic variants in the CLOCK gene were significantly associated with breast cancer risk and CLOCK was overexpressed in breast tumor tissues, suggesting its oncogenic role in breast tumorigenesis (28). As such, hsa-miR-154 is considered as a putative tumor suppressor because it targets the oncogene CLOCK.

The link between hsa-miR-154 and CLOCK has been observed in other human disorders. For example, hsa-miR-154 was upregulated in the prefrontal cortex of people with bipolar disorder (31) and CLOCK gene polymorphism was associated with lifetime recurrence of bipolar episodes (32). Furthermore, hsamiR-154 was found to have a potential role in dopaminergic neuron differentiation and mu-opioid receptor regulation and mutations in the precursor hsa-miR-154 were associated with an addicted phenotype (29). Mice with mutated CLOCK gene have increased cocaine - dopaminergic reward circuitry (30).

Given that hsa-miR-154 is suggested to modulate circadian rhythmicity and mediate post-transcriptional regulation of $C L O C K$ gene, we propose that hsa-miR-154 functions as a tumor suppressor in breast cancer by targeting the CLOCK gene as illustrated in Fig. 4. Moreover, our previous study indicated that night shiftwork related circadian disruption can induce hypermethylation of hsa-miR-154 promoter and may lead to epigenetic silencing of hsa-miR-154 (12). The tumor suppressing role of hsa-miR-154 may provide a new epigenetic mechanism accounting for the observed association between breast cancer and night shiftwork (13). 


\section{Conclusions}

Findings from this study suggest a potential tumor suppressing role of hsa-miR-154 in breast cancer that acts by targeting CLOCK gene. Further investigation of the tumor suppressing role of hsa-miR-154 in more cell lines and in animal models is needed. Confirmation of CLOCK gene as a target of hsa-miR-154 also needs to be validated using experimental assays. Exploration of hsa-miR-154 for its anti-cancer effect may provide information on its potential for therapeutic application.

\section{Declarations}

\section{Ethics approval and consent to participate}

Human data were obtained from public sources.

\section{Consent for publication}

Not applicable.

\section{Availability of data and material}

All data generated or analyzed during this study are included in this published article.

\section{Competing interests}

BEE is a cofounder of Osmol Therapeutics, a company that is targeting NCS1 for therapeutic purposes.

\section{Funding}

This work was partially supported by funds from Yale University and NIH research grant CA238100.

\section{Authors' contributions}

All authors have read and approved the manuscript.

Acquisition of data: $E Y I, A F, A N F$

Aanalyses and interpretation: $\mathrm{EYI}, \mathrm{YZ}$

Writing, review, and/or revision of the manuscript: EYI, BEE, YZ

Conception and design: $\mathrm{YZ}$

\section{Acknowledgements}

Not applicable. 


\section{References}

1. Peng Y, Croce CM. The role of MicroRNAs in human cancer. Signal Transduct Target Ther. 2016;1:15004.

2. Lee J-Y, Ryu D-S, Kim W-J, Kim S-J. Aberrantly expressed microRNAs in the context of bladder tumorigenesis. Investigative and clinical urology. 2016;57(Suppl 1):S52-S9.

3. Nadal E, Zhong J, Lin J, Reddy RM, Ramnath N, Orringer MB, et al. A MicroRNA cluster at 14q32 drives aggressive lung adenocarcinoma. Clinical cancer research. 2014;20(12):3107-17.

4. Uppal A, Wightman SC, Mallon S, Oshima G, Pitroda SP, Zhang Q, et al. 14q32-encoded microRNAs mediate an oligometastatic phenotype. Oncotarget. 2015;6(6):3540.

5. Tian E, Sawyer JR, Heuck CJ, Zhang Q, van Rhee F, Barlogie B, et al. In multiple myeloma, 14q32 translocations are nonrandom chromosomal fusions driving high expression levels of the respective partner genes. Genes, Chromosomes and Cancer. 2014;53(7):549-57.

6. Zhao X, Ji Z, Xie Y, Liu G, Li H. MicroRNA-154 as a prognostic factor in bladder cancer inhibits cellular malignancy by targeting RSF1 and RUNX2. Oncol Rep. 2017;38(5):2727-34.

7. Baranwal S, Alahari SK. miRNA control of tumor cell invasion and metastasis. International journal of cancer. 2010;126(6):1283-90.

8. Mian C, Pennelli G, Fassan M, Balistreri M, Barollo S, Cavedon E, et al. MicroRNA profiles in familial and sporadic medullary thyroid carcinoma: preliminary relationships with RET status and outcome. Thyroid. 2012;22(9):890-6.

9. Qin C, Zhao Y, Gong C, Yang Z. MicroRNA-154/ADAM9 axis inhibits the proliferation, migration and invasion of breast cancer cells. Oncol Lett. 2017;14(6):6969-75.

10. Xu H, Fei D, Zong S, Fan Z. MicroRNA-154 inhibits growth and invasion of breast cancer cells through targeting E2F5. Am J Transl Res. 2016;8(6):2620-30.

11. Tumolo MR, Panico A, De Donno A, Mincarone P, Leo CG, Guarino R, et al. The expression of microRNAs and exposure to environmental contaminants related to human health: a review. Int $\mathrm{J}$ Environ Health Res. 2020:1-23.

12. Shi F, Chen X, Fu A, Hansen J, Stevens R, Tjonneland A, et al. Aberrant DNA methylation of miR-219 promoter in long-term night shiftworkers. Environmental and Molecular Mutagenesis. 2013;54(6):406-13.

13. group IMV. Carcinogenicity of night shift work. Lancet Oncol. 2019;20(8):1058-9.

14. Kadener S, Menet JS, Sugino K, Horwich MD, Weissbein U, Nawathean P, et al. A role for microRNAs in the Drosophila circadian clock. Genes Dev. 2009;23(18):2179-91.

15. Liu C, Kelnar K, Vlassov AV, Brown D, Wang J, Tang DG. Distinct microRNA expression profiles in prostate cancer stem/progenitor cells and tumor-suppressive functions of let-7. Cancer research. 2012;72(13):3393-404.

16. Luo W, Sehgal A. Regulation of circadian behavioral output via a MicroRNA-JAK/STAT circuit. Cell. 2012;148(4):765-79. 
17. Zhu Y, Stevens RG, Leaderer D, Hoffman A, Holford T, Zhang Y, et al. Non-synonymous polymorphisms in the circadian gene NPAS2 and breast cancer risk. Breast Cancer Res Treat. 2008;107(3):421-5.

18. Chung IF, Chang SJ, Chen CY, Liu SH, Li CY, Chan $\mathrm{CH}$, et al. YM500v3: a database for small RNA sequencing in human cancer research. Nucleic Acids Res. 2017;45(D1):D925-d31.

19. Sarver AL, Sarver AE, Yuan C, Subramanian S. OMCD: OncomiR Cancer Database. BMC Cancer. 2018;18(1):1223.

20. Lánczky A, Nagy Á, Bottai G, Munkácsy G, Szabó A, Santarpia L, et al. miRpower: a web-tool to validate survival-associated miRNAs utilizing expression data from 2178 breast cancer patients. Breast Cancer Res Treat. 2016;160(3):439-46.

21. Rao X, Huang X, Zhou Z, Lin X. An improvement of the $2^{\wedge}$ (-delta delta CT) method for quantitative real-time polymerase chain reaction data analysis. Biostat Bioinforma Biomath. 2013;3(3):71-85.

22. Reszka E, Zienolddiny S. Epigenetic Basis of Circadian Rhythm Disruption in Cancer. In: Dumitrescu RG, Verma M, editors. Cancer Epigenetics for Precision Medicine : Methods and Protocols. New York, NY: Springer New York; 2018. p. 173-201.

23. Paraskevopoulou MD, Georgakilas G, Kostoulas N, Vlachos IS, Vergoulis T, Reczko M, et al. DIANAmicroT web server v5.0: service integration into miRNA functional analysis workflows. Nucleic Acids Res. 2013;41(Web Server issue):W169-73.

24. Bandrés E, Cubedo E, Agirre X, Malumbres R, Zárate R, Ramirez N, et al. Identification by Real-time PCR of 13 mature microRNAs differentially expressed in colorectal cancer and non-tumoral tissues. Mol Cancer. 2006;5:29.

25. Maire G, Martin JW, Yoshimoto M, Chilton-MacNeill S, Zielenska M, Squire JA. Analysis of miRNAgene expression-genomic profiles reveals complex mechanisms of microRNA deregulation in osteosarcoma. Cancer Genet. 2011;204(3):138-46.

26. Wang W, Peng B, Wang D, Ma X, Jiang D, Zhao J, et al. Human tumor microRNA signatures derived from large-scale oligonucleotide microarray datasets. Int J Cancer. 2011;129(7):1624-34.

27. Miller BH, McDearmon EL, Panda S, Hayes KR, Zhang J, Andrews JL, et al. Circadian and CLOCKcontrolled regulation of the mouse transcriptome and cell proliferation. Proc Natl Acad Sci U S A. 2007;104(9):3342-7.

28. Hoffman AE, Yi CH, Zheng T, Stevens RG, Leaderer D, Zhang Y, et al. CLOCK in breast tumorigenesis: genetic, epigenetic, and transcriptional profiling analyses. Cancer Res. 2010;70(4):1459-68.

29. Tapocik JD, Luu TV, Mayo CL, Wang BD, Doyle E, Lee AD, et al. Neuroplasticity, axonal guidance and micro-RNA genes are associated with morphine self-administration behavior. Addict Biol. 2013;18(3):480-95.

30. McClung CA, Sidiropoulou K, Vitaterna M, Takahashi JS, White FJ, Cooper DC, et al. Regulation of dopaminergic transmission and cocaine reward by the Clock gene. Proc Natl Acad Sci U S A. 2005;102(26):9377-81. 
Figures

a
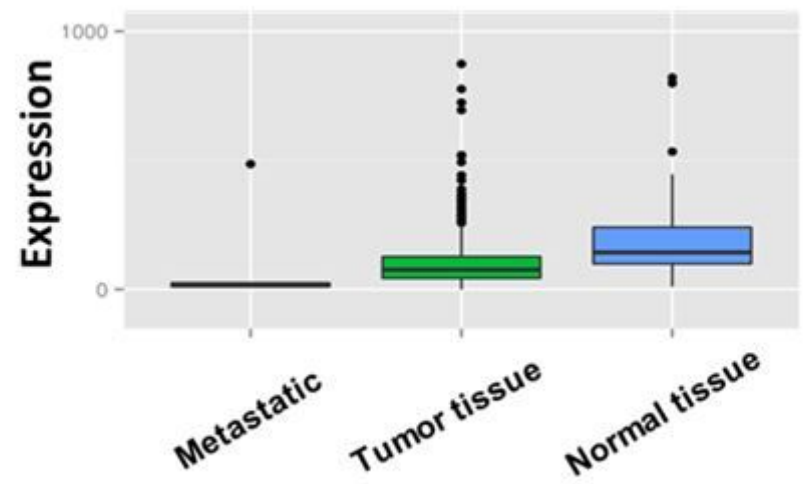

b

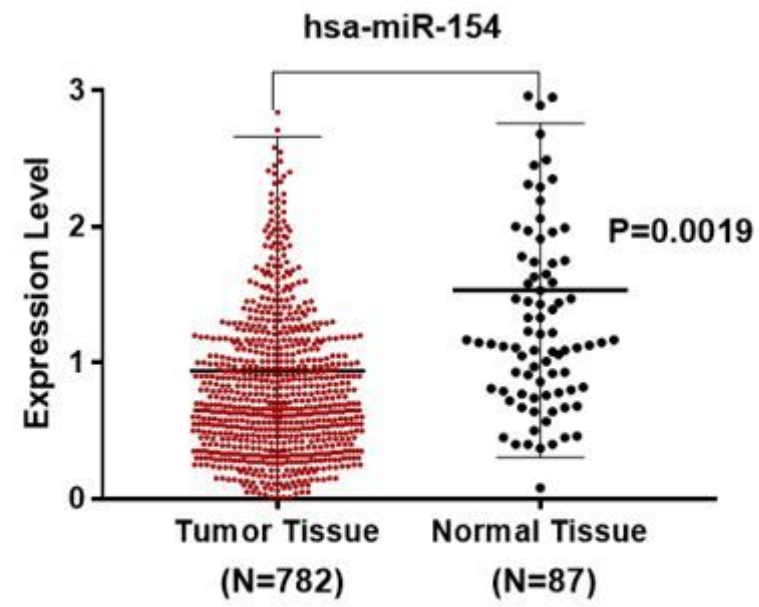

C

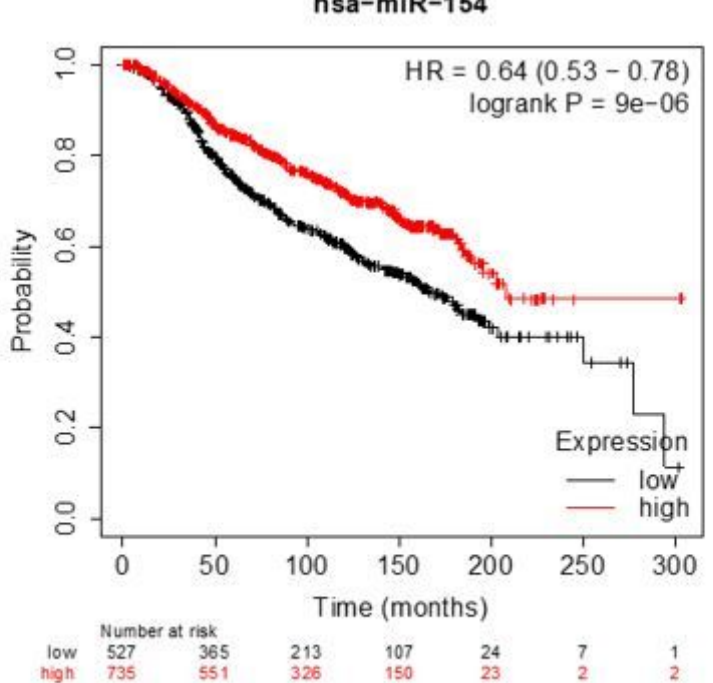

Figure 1

Expression of hsa-miR-154 in breast tumor and its association with breast cancer survival. a) Hsa-miR154 expression in breast cancer tissues (primary, normal and metastatic tissues) using the YM500 database (1006 primary tumors, 103 normal breast tissues, 6 metastatic tissues). The expression is 
downregulated in breast tumor tissues compared to normal breast tissues. b) Expression level of hsamiR-154 significantly decreased in 782 breast cancer tissues in comparison with 87 normal tissues using OncoMir database $(P=0.0019)$. c) Survival analysis of breast cancer patients using the Kaplan Meier plotter (KMplotter) tool, comparing patients with high and low expression of hsa-miR-154. High hsa-miR154 expression correlated with better survival in breast cancer patients ( $P=9.0 \mathrm{e}-6, \mathrm{FDR}: 1 \%$ ). The curve included patients from the METABRIC database with long follow-up (median: 94 months). Hazard ratio with $95 \%$ confidence intervals and logrank $P$ value were calculated and displayed.

a

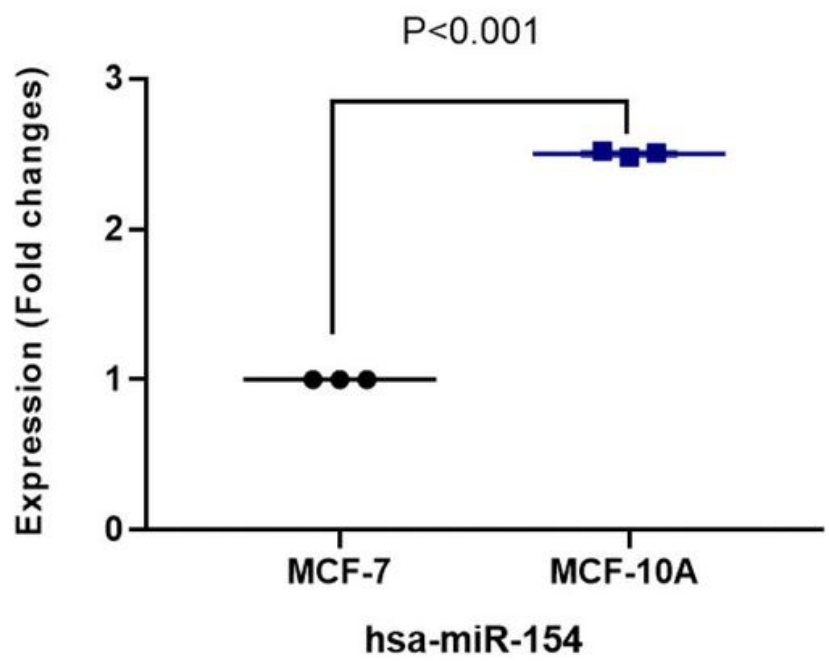

b

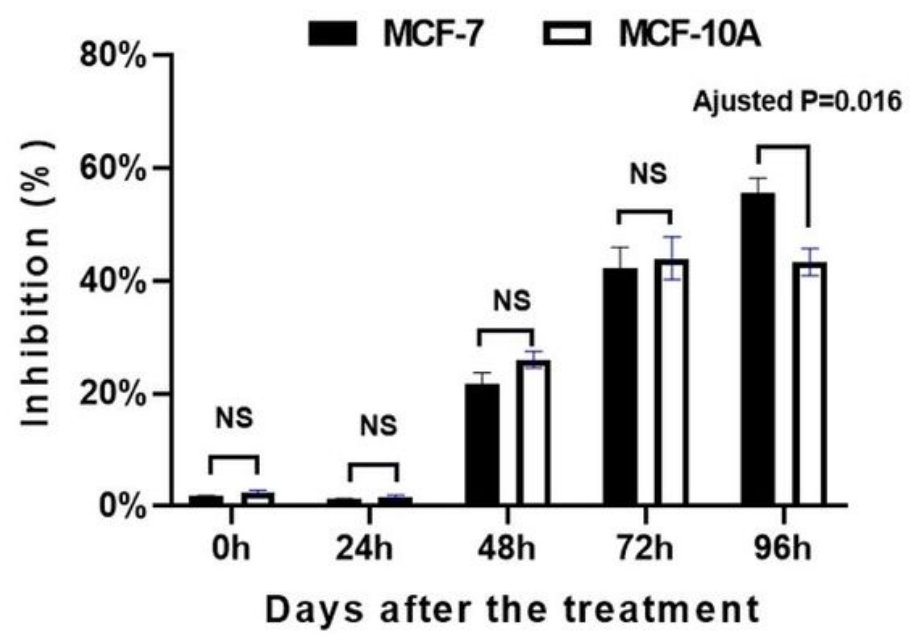

\section{Figure 2}

Restoration of hsa-miR-154 inhibits proliferation of breast cancer cell. a) RT-PCR revealed significantly lower endogenous hsa-miR-154 levels in MCF-7 cells compared to MCF10a cells $(P<0.001)$. b) Hsa-miR154 transfection in both MCF-7 and MCF-10a cell lines significantly inhibited proliferation of breast cancer cells. At 96h, hsa-miR-154 had less effect slowing MCF-10a growth than it did on MCF-7 growth (adjusted $\mathrm{P}=0.016$ after multiple comparison correction). Inhibition was calculated by comparing the proliferation of cells transfected with hsa-miR-154 mimic and cells transfected with negative control. 


\section{a hsa-miR-154}
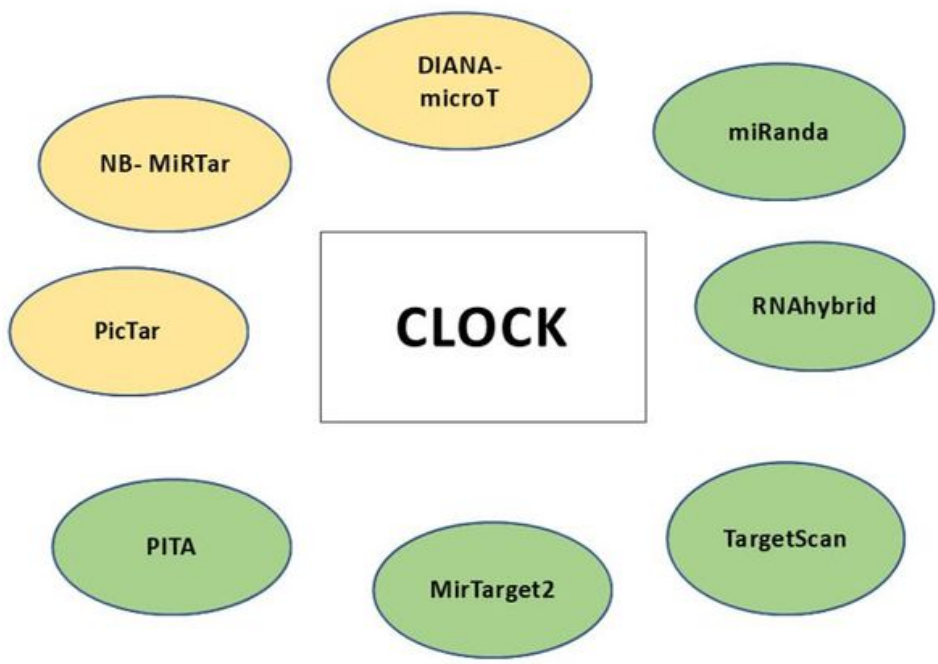

b

$\Delta \mathrm{G}=-19.1 \mathrm{kcal} / \mathrm{mol}$
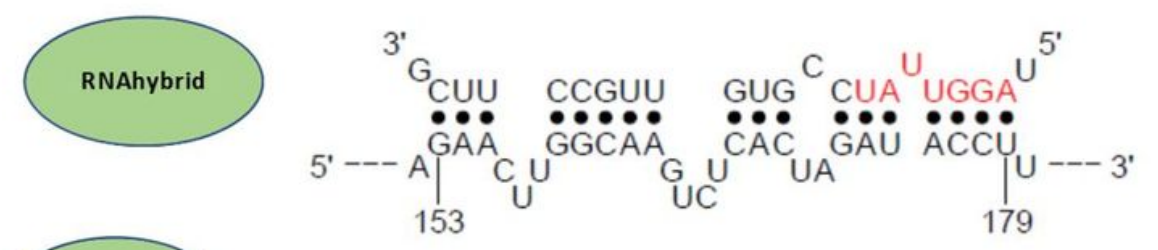

NM_004898

\section{Figure 3}

Circadian CLOCK gene is a potential target of hsa-miR-154. a) Potential hsa-miR-154 targets of CLOCK gene using several prediction tools. Targets detected using the tools are colored green and those not detected are colored yellow. b) Potential hsa-miR-154 sequence match of CLOCK gene (highlighted in red) using the IntaRNA prediction tools.

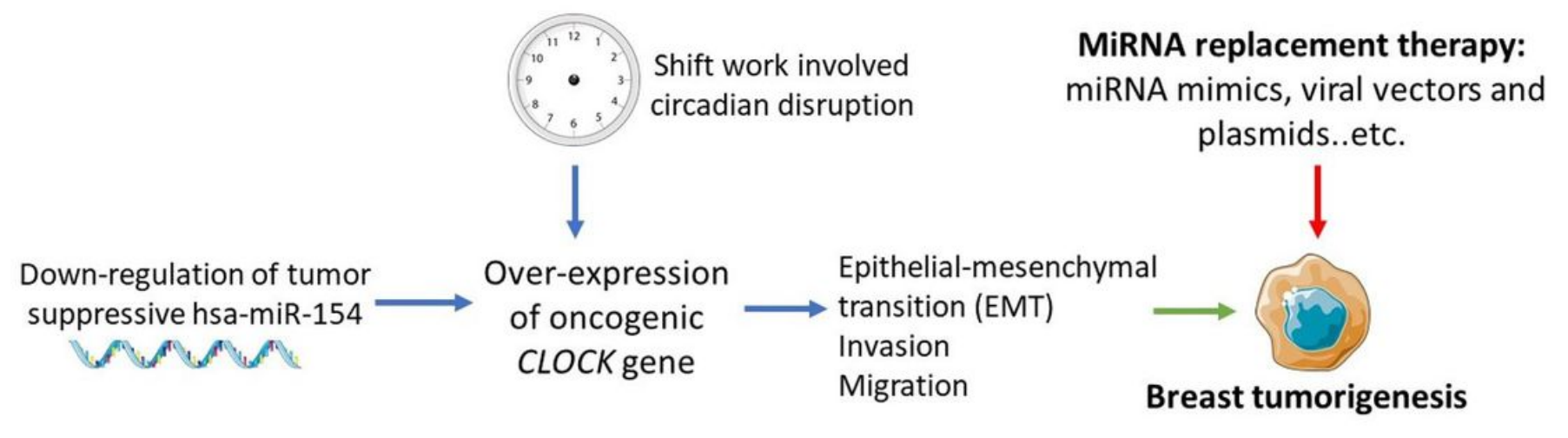

\section{Figure 4}

A schematic model of the proposed roles of hsa-miRNA-154 involved in breast tumorigenesis. This figure was produced using Servier Medical Arts (https://smart.servier.com). 